Original Research Paper

\title{
Effect of Stevia Consumption on Blood Pressure, Stress Hormone Levels and Anthropometrical Parameters in Healthy Persons
}

\author{
${ }^{1}$ Emad A.S. Al-Dujaili, ${ }^{2}$ Husni Twaij, ${ }^{2}$ Yazan A. Bataineh, ${ }^{1}$ Unam Arshad and ${ }^{1}$ Faiza Amjid \\ ${ }^{1}$ Queen Margaret University, Dietetics, Nutrition and Biological Sciences, Edinburgh, UK \\ ${ }^{2}$ Department of Pharmacology, Philadelphia University, Amman Jordan
}

\author{
Article history \\ Received: 19-03-2017 \\ Revised: 28-03-2017 \\ Accepted: 29-03-2017 \\ Corresponding Author: \\ Emad A.S. Al-Dujaili \\ Queen Margaret University, \\ Edinburgh, UK \\ Email: ealdujaili@qmu.ac.uk \\ Or ealdujai@staffmail.ed.ac.uk
}

\begin{abstract}
Stevia is a natural sweetener containing steviol glycosides known to be several times sweeter than sucrose. It is thought to have several beneficial properties though some evidence state it may have detrimental effects. The aim of this study was to investigate the potential beneficial or harmful effects of stevia consumption by exploring its effects on blood pressure, stress hormone levels and anthropometrical markers in A crossover placebo controlled study was conducted on 16 volunteers randomly assigned to consume either stevia or a placebo (sugar) for one week. The measurements were attained on three different occasions and each volunteer was allowed a 3-day initiation period before baseline and in between interventions. The systolic BP increased following stevia intake from $114.5 \pm 12.7$ to $119.9 \pm 12.9 \mathrm{mmHg}(\mathrm{p}<0.001)$ and diastolic BP from $70.8 \pm 9.4$ to $75.7 \pm 9.6 \mathrm{mmHg}(\mathrm{p}<0.01)$. Systolic BP increased slightly after the sugar placebo to $115.3 \pm 13.6 \mathrm{mmHg}$ (not significant). The mean free cortisol excreted in urine has increased from $91.8 \pm 49.1$ to $125.7 \pm 60.5 \mathrm{nmole} /$ day $\quad(\mathrm{p}<0.01)$ after the stevia and to $109.1 \pm 42.6 \mathrm{nmole} /$ day after the placebo $(\mathrm{p}=0.210)$. The ratio of urinary free cortisol/cortisone showed a statistically significant increase from $1.73 \pm 0.78$ to $2.65 \pm 1.03$ after stevia $(\mathrm{p}<0.0001)$. Salivary cortisol levels have also increased $(\mathrm{p}<0.01$ at $\mathrm{AM})$ after stevia. Placebo intake did not produce a significant change in salivary cortisol. The ratio of salivary cortisol/cortisone during the stevia has increased only in the morning (from $1.22 \pm 0.65$ to $1.75 \pm 0.72, \mathrm{p}=0.05$ ) and a modest increase in the daily average of salivary cortisol/cortisone. There was small insignificant reduction in weight and $B M I$ after stevia intervention $(p=0.246$ and $p$ $=0.249$ respectively). In conclusion, we have shown that short term stevia intake produced a small but significant increase in BP and effect on body weight and BMI were not significant. The rise in BP might be due to the increase in cortisol levels and cortisol/cortisone ratio indicating that stevia may possibly inhibit $11 \beta$-HSD2 enzyme by reducing the conversion of cortisol into cortisone. Therefore caution should be taken by the public who want to consume stevia for longer period of time as a weight reducing sweetener.
\end{abstract}

Keywords: Stevia, Sweeteners, Blood Pressure, BMI, Glucocorticoids, $11 \beta$-HSD

\section{Introduction}

Stevia is a sweetener and a natural herb that comes from the Asteraceae family originating from South America that is several times sweeter than sucrose (cane sugar) and has previously been used in traditional medicine (Giuffré et al., 2013; Sorensen et al., 2014). Currently it is used in some countries as a substitute for sugar by overweight, obese and diabetic patients (Hwang et al., 2007; Goyal and Samsher, 2010; Shivanna et al., 2013). 
Several active stevioside glycosides metabolites (Gardana et al., 2003; Geun et al., 2007) have been found in the leaves of stevia including: Isosteviol, stevioside, rebaudiosides $A$ to $F$, steviolbioside and dulcoside; though stevioside and rebaudioside A are the focal metabolites that are the most sweet, heat stable, $\mathrm{pH}$ stable and nonfermentable compounds (Khalil et al., 2014; Gupta et al., 2013). Stevia leaves are thought to possess useful pharmaceutical properties (Brahmachari et al., 2011) and several studies have reported a number of health benefits associated with stevia consumption. It has been found that the leaves of $S$. Rebaudiana have medicinal properties; antimicrobial, antiviral, antifungal, antihypertensive, anti-hyperglycaemic, anti-inflammatory, anti-tumour and diuretic effects (Wheeler et al., 2008; Roberts and Munro, 2009; Gheisar and Kim, 2014; Onakpoya and Heneghan, 2015). Toxicological reports have also shown that secondary metabolites present in stevia did not have teratogenic, mutagenic (Lemus-Mondaca et al., 2012) or carcinogenic effects. No allergic reactions have been detected following stevia consumption as a sweetener (Carakostas et al., 2008; Ferri et al., 2006; Gupta et al., 2013).

In recent years obesity has become a dilemma internationally and a noticeable factor that influences the increase incidence of obesity is the excess consumption of foods and drinks that contain a great amount of refined sugars and calories (Bryant et al., 2014). Stevia has been found to contain no calories or carbohydrates (Ahmed et al., 2011) and thus received some attention by the general public and has become increasingly popular as a sweetener. Therefore it has been suggested by some researchers that stevia being a natural herb could be a successful method to control weight gain if it replaces sugar in the diet (Anton et al., 2010; Yadav and Guleria, 2012; Gupta et al., 2013). A study on rats seemed to show a decrease in body weight when stevia was orally ingested (Curry and Roberts, 2008). Additionally it has also been suggested that stevia might have an anti-hyperglycaemic effect (Gregersen et al., 2004; Jeppesen et al., 2006) and a recent study concluded that there was a significant reduction in blood glucose levels in rats after stevioside intake (Rizzo et al., 2013). Stevioside and steviol have also been shown to have a direct effect on the beta cells of the pancreas to stimulate the secretion of insulin and improve glucose tolerance that might prove to have a positive impact in the prevention of type 2 diabetes mellitus (Sattigeri, 2012). However, Ferri et al. (2006) and others (Chan et al., 2000; Hsieh et al., 2003) reported no significant effect of stevia on glucose homoestasis.

Hypertension occurs when both systolic and diastolic blood pressure in the arteries become elevated persistently at or above $140 \mathrm{mmHg}$ systolic and $90 \mathrm{mmHg}$ diastolic (Whitworth, 2003). Many studies have shown that stevia can be used to decrease Blood Pressure (BP), both systolic and diastolic (Chan et al., 2000; Ranjan et al., 2011). However, the reduction was very small and not significant in systolic BP according to a recent systematic review and meta-analysis of randomised clinical trials, but there was a significant reduction in diastolic BP and fasting blood glucose (Onakpoya and Heneghan, 2015). On the other hand, another systematic review found that the effects of stevia on blood pressure were debatable as the results were diverse and inconclusive as far as the increase or decrease in blood pressure depending on the duration of study and participating subjects (Ulbricht et al., 2010). The review has also found that in studies performed for a period of 1 to 3 months, stevia increased blood pressure. Therefore, it is apparent that this subject remains controversial and requires further studies. An interesting study presented a case report that showed a middle aged woman who suffered from oedema, hypocalcaemia and prehypertension brought on by inhibiting the enzymeHSD2 (11ß-Hydroxysteroid dehydrogenase 2) which was caused by the consumption of stevia over a long period of time. It was found that the daily consumption of stevia as a sweetener could lead to an increase in blood pressure by reducing the amount of cortisol that was converted into cortisone through the inhibition of $11 \beta$-HSD2 and therefore enhancing the activity of cortisol in the kidney nephrons to reabsorb sodium and water (Esmail and Kabadi, 2012).

The effect of stevia on the levels of cortisol and cortisone after stevia consumption was another aim of this study. Naturally produced by the body, cortisol is a glucocorticoid hormone also known as the stress hormone where the active form is cortisol and the inactive form is cortisone. The hormone released in response to stress and low blood sugar levels, serves to control inflammation by suppressing some of the immune system inflammatory markers and exerts a variety of metabolic functions. Cortisone (the inactive form) is converted to the active form, cortisol by the

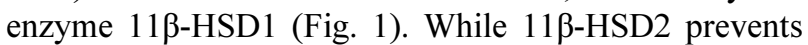
overstimulation of the mineral corticoid receptor by cortisol that works to increase the active steroid levels in responsive tissues (Quinkler and Stewart, 2013). Studies have also seemed to show that stevia increases levels of cortisol in the body by suppressing $11 \beta$-HSD2 in human participants (Esmail and Kabadi, 2012), yet some other studies have reported that stevia consumption by participants for over a month did not produce any considerable effect on cortisol and cortisone levels (Corcuff and Brossaud, 2014). Therefore, there seems to be controversial data on the effect of stevia on glucocorticoids levels. 


\section{$11 \beta$-hydroxysteroid dehydrogenase interconverts active and inactive glucocorticoids}
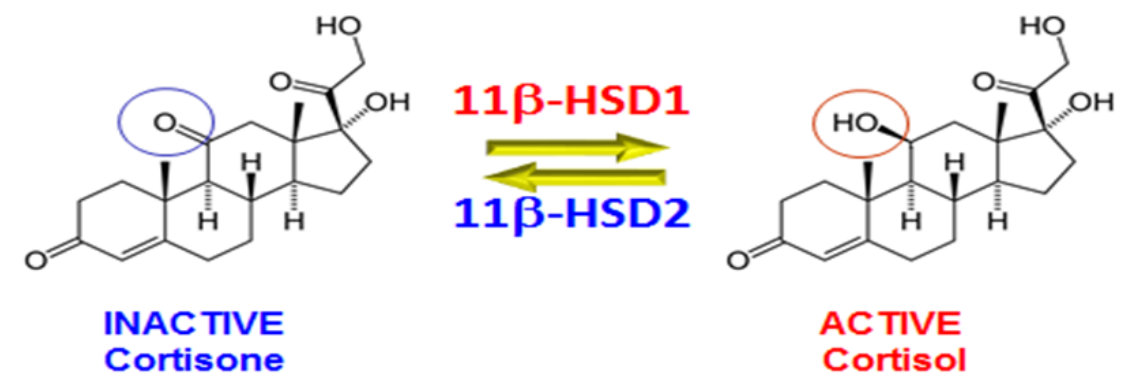

Fig. 1. Inter-conversion of inactive cortisone and active cortisol by $11 \beta$-HSD enzymes (Al-Dujaili et al., 2011)

\section{Materials and Methods}

\section{Study Population}

For this project, a total of 16 volunteers who met the criteria for this study and were eligible to take part recruited from Queen Margaret University's staff and student population through the QMU moderator email as well as the general public out with the University. The necessary criteria that had to be attained by volunteers for this project were evaluated through a health status questionnaire in which male or female subjects with a wide range of BMI were included. Volunteers were included between the ages of 19 to 60 years. The inclusion criteria were male and females who were apparently healthy, non-smokers, not diabetics and not on medications that lower BP. The exclusion criteria involved individuals who were under the age of 18 or above 65 with a medical history of CVD including hypertension and diabetes mellitus. In addition, volunteers who smoke were excluded from the study as smoking may potentially have an effect on blood pressure changes. The 16 volunteers that agreed to participate in the study were provided with an information sheet and consent forms that were completed by all participants. The samples and data collected from all volunteers had been kept anonymous by replacing their names with identification numbers. The study procedure and ethical approval was granted by Queen Margaret University's Research Ethics Committee.

\section{Study Design}

This project adopted a randomised cross over placebo controlled study where each volunteer was given a code number for identification. Measurements on each volunteer were taken three times; at baseline, after stevia or the placebo intervention. The volunteers taking part in this study were randomly divided into two groups and both groups started with a
3 day washout period, followed before and after each intervention to prevent a carryover effect. The first group were allocated stevia to be taken for 7 days and the second group were allocated the placebo (sucrose, table sugar) to be taken for 7 days and were given specific instructions not to take any additional or excess amounts of sugar in their diet during this period. Figure 2 illustrates the design of the crossover study for this project.

\section{Sample Collection}

At the start of the project each volunteer was asked to provide a $24 \mathrm{~h}$ urine collection and 3 saliva (morning, afternoon and evening) samples as a baseline measurement. The data was necessary for the repeated measure statistics and comparison between stevia and the placebo. After the 3 days initiation period each participant was given either $5 \mathrm{~g}$ of sugar or $0.2 \mathrm{~g}$ of stevia to consume 3 times a day for 1 week. On the $7^{\text {th }}$ day of the intervention, each participant was required to collect a $24 \mathrm{~h}$ urine sample along with 3 saliva samples; morning, afternoon and evening. This process was repeated for another week when participants crossed over to take the opposite intervention and samples were once again collected on the $7^{\text {th }}$ day.

\section{Intervention Diet}

In this study, $100 \%$ pure natural stevia was used in the form of powder and purchased from Boots Ltd, UK. The placebo used in this study was Tate Lyle table sugar. Volunteers were asked to consume the stevia or sugar 3 times a day, preferably in a hot drink of their choice. Volunteers were instructed to refrain from consuming any other forms of sweeteners and sugar, throughout the duration of the study. The dose of stevia consumed by our participants was chosen to simulate the routine use of stevia as a substitute for sugar in their daily life and not to evoke any unwanted side effects. 


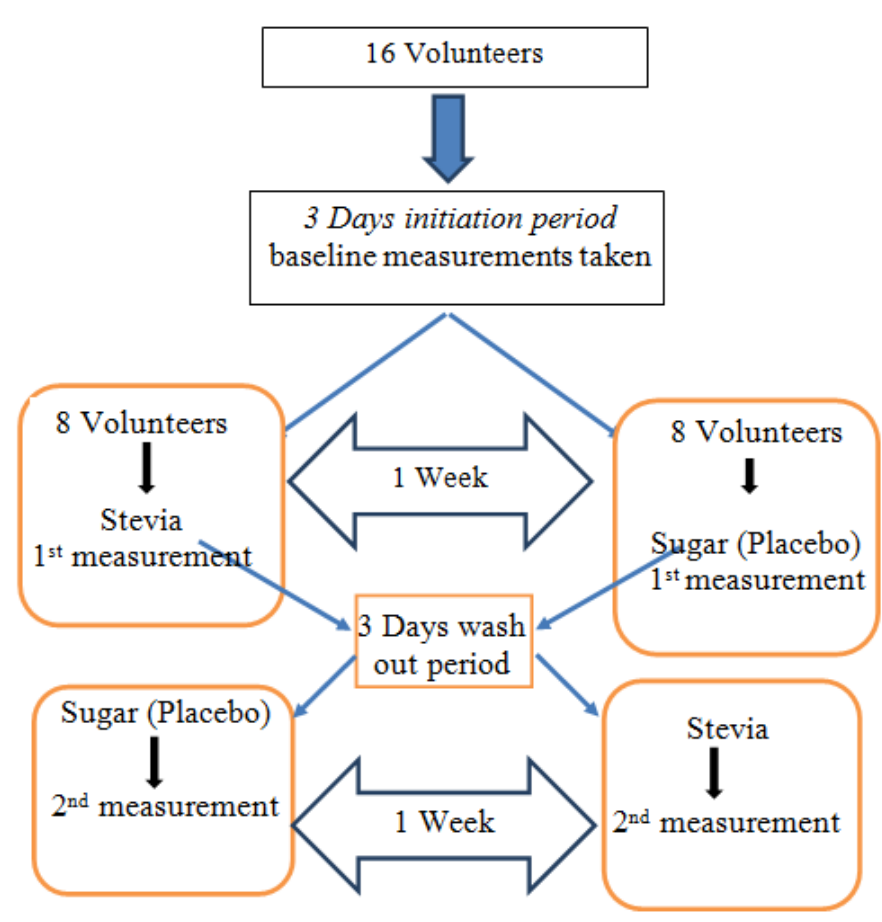

Fig. 2. Crossover design of the study showing the protocol each participant has to follow during the entire study. A 3-day wash out period was chosen due to time restriction and subject convenience

\section{Measurements}

Physiological variables that were measured in this study alongside the biomarkers in urine and saliva were blood pressure, weight, height and BMI. To measure BP, a digital sphygmomanometer was used while participants were seated comfortably and allowed to relax for 5 to 10 min to avoid "white coat" hypertension (Franklin et al., 2013). BP was taken three times with 5-minute intervals in between, with the mean calculated and used. Weight and height were also measured to obtain each volunteer BMI score before and after each intervention. The height of each volunteer was measured by a leister-height scale and their weight was measured by the same digital scale every time to prevent inaccuracies. The BMI of each volunteer was calculated by the following equation: BMI $\left(\mathrm{kg} / \mathrm{m}^{2}\right)=$ weight $(\mathrm{kg}) /$ height $^{2}(\mathrm{~m})$. The urine and saliva samples collected were weighed and stored in the freezer at $-20^{\circ} \mathrm{C}$ in small sample tubes, to avoid any fungal or bacterial growth. Cortisol and cortisone in saliva samples were then analysed by the Enzyme-Linked Immunosorbent Assay (ELISA) technique using Salimetric kits (USA). For urine samples, an in-house indirect ELISA method was used prior to solvent extraction (Al-Dujaili et al., 2012).

\section{Statistical Analyses}

A parametric test was carried out when all variables had been assessed for normal distribution. To evaluate the variation between the three interventions; basal, stevia and placebo, one way ANOVA was performed. Post hock comparisons using Bonferroni's method was used to see which groups were statistically different (Pallant, 2001). Since not all parameters were normally distributed the ANOVA reading might be biased and thus, a student 2-tailpaired t-test was also performed to compare differences between the baseline and the two interventions; stevia and placebo. This method was carried out using SPSS statistics (version17.0) and Microsoft Word Excel 2010. All results presented as the mean \pm SD or SEM and significance by the $p$ value $\leq 0.05$ (Bland, 2015; Field, 2005).

\section{Results}

\section{Subject Characteristics}

In this study 16 healthy volunteers participated ( 8 males and 8 females) with an age range of 18-60 years and mean \pm SD of $27.75 \pm 13.75$ years and BMI of the participants ranged from $20.6-36.4 \mathrm{~kg} / \mathrm{m}^{2}$ and mean of $26.33 \pm 5.26 \mathrm{~kg} / \mathrm{m}^{2}$. Table 1 shows the characteristics and demographics of subjects participating in the study. Six participants were regular coffee and tea drinkers consuming between 2-4 cups a day, whereas 10 people were not. Three participants were taking protein shakes and all female participants were not on any type of contraceptive drugs. All participants did regular exercise of at least $30 \mathrm{~min}$ of walking per day. 


\section{Effect on Blood Pressure}

Basal Blood pressure measurements of participants were attained after the first 3 days initiation period and the mean $\pm \mathrm{SD}$ values were as follows; systolic BP was $114.5 \pm 12.7 \mathrm{mmHg}$ and diastolic BP $70.8 \pm 9.4 \mathrm{mmHg}$. The systolic BP increased to $119.9 \pm 12.9 \mathrm{mmHg}(\mathrm{p}<0.001)$ after the stevia intervention and increased slightly again after the placebo sugar intervention to $115.3 \pm 13.6 \mathrm{mmHg}$. However this increase was found to be statistically insignificant $(p=0.685)$. All the parameters measured were presented as mean \pm SD in Table 2. For diastolic blood pressure, there was also a significant increase from basal $70.8 \pm 9.4$ to $75.7 \pm 9.6 \mathrm{mmHg}$ after stevia intervention $(p<0.01)$, but there was no significant change after placebo intervention (Fig. 3A). Also there was a statistically significant difference when comparing BP following stevia intake with placebo intervention $(\mathrm{p}=0.01$ and $\mathrm{p}=$ 0.044 for SBP and DBP respectively).

\section{Effect on Anthropometrical Parameters}

At basal measurements the participant's weight and BMI mean values were $74.82 \pm 16.5 \mathrm{~kg}$ and $26.33 \pm 5.2 \mathrm{~kg} / \mathrm{m}^{2}$ respectively. There was no significant reduction found in weight or BMI after stevia intervention $(\mathrm{p}=0.246$ and $\mathrm{p}=$ 0.249 respectively), yet there was a slight decrease shown in both parameters (mean of $74.2 \pm 16.1 \mathrm{~kg}$ and $26.1 \pm 5.1$ $\mathrm{kg} / \mathrm{m}^{2}$ ). Again no significant changes were found after placebo intervention with $\mathrm{p}=0.787$ for weight and $\mathrm{p}=$ 0.796 for BMI, but mean values showed a very slight increase at $75.1 \pm 16.6 \mathrm{~kg}$ for weight and $26.4 \pm 5.3 \mathrm{~kg} / \mathrm{m}^{2}$ for BMI. When comparing both interventions similarly no significant changes were found with $p=0.242$ for weight and $\mathrm{p}=0.227$ for BMI (Table 2).

\section{Effect on Urinary and Salivary Cortisol and Cortisone levels}

The mean basal levels excreted in urine were as follows (Fig. 3B); free cortisol was $91.8 \pm 49.1 \mathrm{nmole} /$ day and free cortisone 57.3 nmole/day. Cortisol daily excretion has increased in the urine to $125.7 \pm 60.5$ nmole/day $(p<0.01)$ after the stevia intervention and to $109.1 \pm 42.6$ nmole/day after the placebo intervention which was not significant $(\mathrm{p}=$ $0.210)$. There was also no statistical significance found when comparing the cortisol concentration after stevia and placebo intervention $(\mathrm{p}=0.243)$. Urinary free cortisone also slightly decreased after stevia intervention to $49.5 \pm 20.6$ nmole/day $(\mathrm{p}=0.02)$ and after placebo intervention to 55.6 nmole/day, however this was not statistically significant ( $p$ $=0.703$ ). Most importantly, the ratio of excreted urinary free cortisol over free cortisone showed a statistically significant increase from $1.73 \pm 0.78$ at basal to $2.65 \pm 1.03$ after stevia $(p<0.0001)$ or placebo intervention to $2.08 \pm 0.71$ which was just not significant ( $p=0.07$, Table 2).

Salivary cortisol and cortisone showed an expected good circadian rhythm; Fig. 3C and 3D demonstrate the salivary daily rhythm for cortisol and cortisone at basal levels and following the consumption of stevia or placebo for one week. For cortisol, there was a rise in the salivary levels at all 3 points but only in the morning, the increase was significant $(p<0.01)$ after stevia. Placebo intake did not produce a significant change in salivary cortisol. For Cortisone, stevia did not affect significantly cortisone circadian rhythm. However, intake of placebo was found to increase noon saliva cortisone significantly $(p=0.01)$. The ratio of cortisol/cortisone during stevia was increased only at the morning time (from $1.22 \pm 0.65$ to $1.75 \pm 0.72, \mathrm{p}=$ 0.05 ) compared to basal ratio, but was not significant at noon and evening times. Following placebo, there was a significant decrease in the ratio only at the morning time (from $1.22 \pm 0.65$ to $1.06 \pm 048, \mathrm{p}=0.01$ ). The overall mean basal cortisol concentration found in saliva calculated by averaging the level at am, noon and pm has increased from $5.58 \pm 2.5$ to $6.77 \pm 3.1$ nmole $(\mathrm{p}<0.01)$ following stevia, however no significant increase was obtained after the placebo $(p=0.09$, Table 2$)$. Also there was no significant change observed in salivary cortisone levels after stevia or placebo (Table 2). The ratio of cortisol/cortisone average saliva output $(\mathrm{am}+$ noon $+\mathrm{pm})$ was increased after stevia which was just significant $(\mathrm{p}=0.050)$, but again no statistical significance was found between the ratio at average basal and after placebo $(p=0.371$, Table 2$)$.

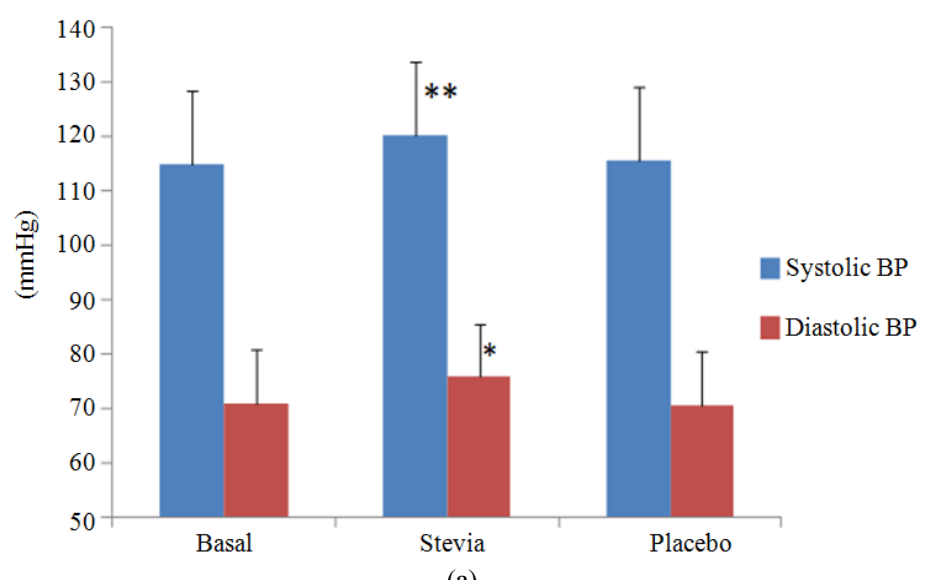

(a) 


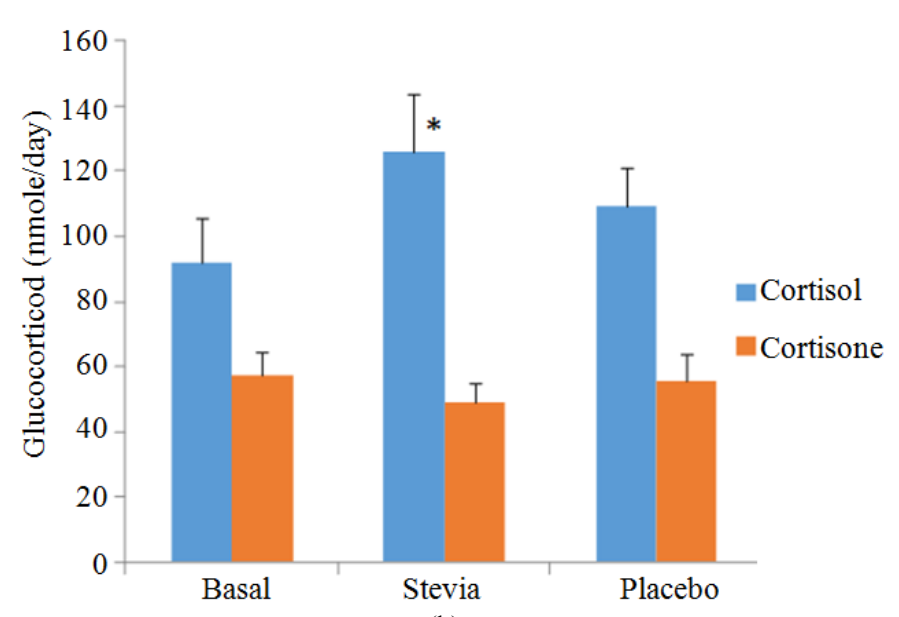

(b)
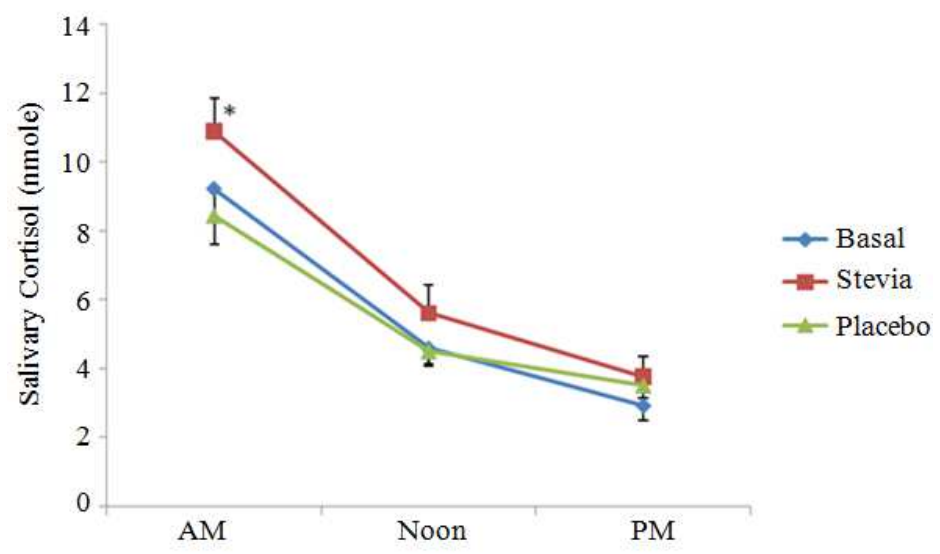

(c)

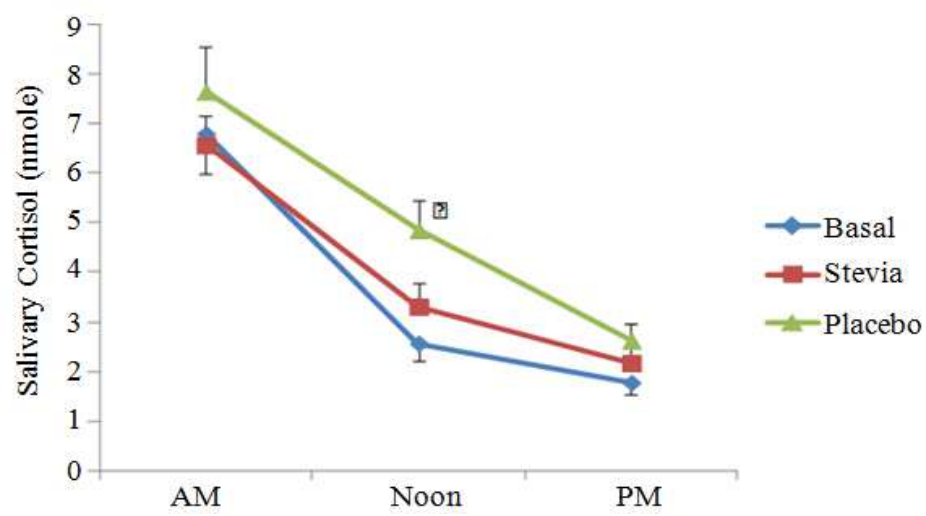

(d)

Fig. 3. Effects of stevia consumption on physiological and biochemical markers in healthy volunteers: (a) Systolic and Diastolic BP (mean $\pm \mathrm{SD}$ ) at basal, after stevia or placebo intervention. SBP and DBP increased modestly but significantly after stevia compared to basal levels. No significant change was observed after the placebo, (b) Urinary Free Glucocorticoids excretion. Free cortisol daily excretion has increased significantly after the stevia compared to basal levels. No significant change in free cortisone excretion and nor following the placebo were observed. (c) Salivary cortisol circadian rhythm (mean \pm sem). There was a rise in salivary cortisol levels at all 3 points after stevia but only at the AM period, the increase was significant. There was no significant difference between placebo and basal salivary levels, (d) Salivary Cortisone circadian rhythm (mean \pm sem). Stevia did not affect significantly cortisone circadian rhythm levels. However, intake of placebo has increased slightly salivary cortisone levels significantly at noon compared to stevia intake ${ }^{*} \mathrm{p}<0.001,{ }^{*} \mathrm{p}<0.01$ and $\square \mathrm{p}=0.01$ 
Table 1. Characteristics and demographics of subjects participating in the study

\begin{tabular}{llll}
\hline Characteristic & Male & Female & Overall \\
\hline Gender & 8 & 8 & 16 \\
Age (year) & $26.2 \pm 9.7$ & $30.3 \pm 15.6$ & $27.75 \pm 13.75$ \\
Weight $(\mathrm{kg})$ & $78.53 \pm 19.8$ & $71.11 \pm 13.4$ & $74.82 \pm 16.59$ \\
Height $(\mathrm{m})$ & $1.69 \pm 0.112$ & $1.677 \pm 0.096$ & $1.68 \pm 0.11$ \\
BMI $\left(\mathrm{kg} / \mathrm{m}^{2}\right)$ & $27.43 \pm 6.56$ & $25.23 \pm 3.8$ & $26.33 \pm 5.26$ \\
SBP $(\mathrm{mmHg})$ & $114.9 \pm 13.6$ & $114.3 \pm 12.1$ & $114.5 \pm 12.7$ \\
DBP $(\mathrm{mmHg})$ & $73.5 \pm 8.7$ & $68.2 \pm 7.9$ & $70.8 \pm 9.4$ \\
Coffee/tea pre-study intake (n) & 3 & 3 & 6 \\
Ethinicity origin & & 3 & 7 \\
(a) Scottish & 4 & 5 & 9 \\
(b) Asian & 4 & & \\
\hline
\end{tabular}

Table 2. Population characteristics and effect of intervention on physiological, anthropometry parameters and stress hormones

\begin{tabular}{llll}
\hline Volunteers parameters & Baseline Mean \pm SD & Stevia Mean \pm SD & Placebo Mean \pm SD \\
\hline Weight $(\mathrm{kg})$ & $74.82 \pm 16.5$ & $74.2 \pm 16.1$ & $75.1 \pm 16.6$ \\
BMI $\left(\mathrm{kg} / \mathrm{m}^{2}\right)$ & $26.33 \pm 5.2$ & $26.1 \pm 5.1$ & $26.4 \pm 5.3$ \\
Systolic BP $(\mathrm{mmHg})$ & $114.5 \pm 12.7$ & $119.9 \pm 12.9^{* *}$ & $115.3 \pm 13.6$ \\
Diastolic BP (mmHg) & $70.8 \pm 9.4$ & $75.7 \pm 9.6^{*}$ & $70.9 \pm 11.9$ \\
Average saliva Cortisone at am, noon and pm (nmole) & $3.72 \pm 1.7$ & $3.64 \pm 1.4$ & $3.9 \pm 1.5$ \\
Average saliva Cortisol at am, noon and pm (nmole) & $5.58 \pm 2.5$ & $6.77 \pm 3.1^{*}$ & $5.5 \pm 2.3$ \\
Ratio of average Saliva Cortisol/cortisone at am, noon and pm & $1.68 \pm 0.77$ & $1.98 \pm 0.84 \#$ & $1.64 \pm 0.68$ \\
Ratio Urinary excretion of Cortisol/cortisone & $1.73 \pm 0.78$ & $2.65 \pm 1.03^{* * *}$ & $2.08 \pm 0.75$ \\
\hline
\end{tabular}

$* * * \mathrm{p}<0.0001, * * \mathrm{p}<0.001, * \mathrm{p}<0.01, \# \mathrm{p}=0.05$

\section{Discussion}

The important finding of this study was that the consumption of stevia for one week did slightly increase systolic and diastolic BP and such increase was statistically significant. However, placebo did not change systolic and diastolic BP significantly. Additionally, this study demonstrated that the consumption of stevia, even though did not lead to a significant reduction in body weight, did show a trend for possible loss in body weight bearing in mind the short duration of the study. The findings from the analysis of the participants' urine and saliva samples at basal and post interventions revealed a significant increase in salivary and urinary free cortisol levels. Cortisone levels however were not much affected. The ratio of cortisol/cortisone which is an indicative marker of the activity of $11 \beta$-HSD enzymes has significantly increased following stevia intake. The increase in both systolic and diastolic BP was corroborated in the case study by Esmail and Kabadi (2012) that presented a middle aged woman with oedema, prehypertension and hypocalcaemia who consumed stevia regularly for 9 months who showed an increase in blood pressure that resulted in her developing hypertension. Laboratory tests also found that there was an increase in plasma cortisol/cortisone ratio and seemed to conclude that a reduced rate of conversion of cortisol into cortisone by inhibiting $11 \beta-H S D 2$ enzyme. This was again investigated in a systematic review which concluded that studies performed for 1-3 months found stevia intake produced an increase in blood pressure, but there was lowering of $\mathrm{BP}$ in hypertensive patients consuming stevia for longer periods of 1-2 years
(Ulbricht et al., 2010). In contrast to our findings, other studies have found stevia extract intake or its isolated glycosides has contributed to healthy blood pressure regulation by inducing vaso-relaxation in a 2-year study using a very high dose of $1500 \mathrm{mg}$ stevioside daily (Gupta et al., 2013). The anti-hypertensive effects of stevia have been also reported by Ahmed et al. (2011) and another study showed a decrease in systolic and diastolic BP in participants with mild hypertension using a high dose of $750 \mathrm{mg}$ stevia (Thomas and Glade, 2010). Therefore, it seems that the latter studies have used much larger doses than our study which might have caused some side effects and interfere in other mechanisms that modulate $\mathrm{BP}$ on the other hand, several researchers have found no effects of stevia consumption on blood pressure (Ferri et al., 2006; Barriocanal et al., 2008; Maki et al., 2008).

Stevia consumption was found to increase cortisol levels significantly in this study in both urine and saliva samples. However the mean values of cortisone were not changed significantly. However another study has shown that stevia consumption over one month did not produce any considerable effect on cortisol and cortisone levels (Corcuff and Brossaud, 2014). Esmail and Kabadi (2012) found that the daily consumption of stevia over a period of 9 months showed an increase in plasma cortisol/cortisone ratio due to a reduction in the amount of cortisol getting converted into cortisone as there was an inhibition of $11 \beta$ HSD2 enzyme in agreement with our findings. The connection between cortisol and cortisone levels and blood pressure and hypertension has been recognized to occur either with a deficit of $11 \beta$-HSD2 as a congenital defect or inhibited by the chronic consumption of liquorice 
(Stewart et al., 1990; Edwards, 1991). Liquorice and stevia seem to have similar effects in regards to oral consumption and their effects on both BP and glucocorticoid levels (Al-Dujaili et al., 2011). Other researchers have also investigated the effects of rebaudioside $\mathrm{A}$ on the excretion of cortisol metabolites in 23 healthy subjects consuming 4 pellets of a sweetener containing stevia three times per day (Corcuff and Brossaud, 2014). They suggested that intake of stevia extracts had no significant modification of the ratios of free cortisol/cortisone or their metabolites. However, they concluded that further research would be needed since their study was short term and used a particular rebaudioside A product and stevia extract powder could be a mixture of molecules that can be extremely variable. Further investigations were required to establish the long term effects of stevia on the cortisone/cortisol ratio and blood pressure. The production of cortisol has been known to be in response to stress and cortisol is an essential hormone that regulates several physiological and metabolic processes (Delaney, 2014) and in particular, in type 2 diabetes and metabolic syndrome where enzyme activities may be modified and superimposed rebaudioside effect could act differently in these conditions (Pereira et al., 2012). In addition, high levels of cortisol could result in a reduced sensitivity to insulin and eventually leading to cardiovascular damage and Cushing's syndrome (NHS Choice, 2013).

There was a very small reduction in body weight and BMI seen in this study though statistically insignificant which might be due to the short duration of the study. A study by Curry and Roberts (2008) found that a decrease in body weight in rats when stevia was orally ingested. Consumption of stevia was also found to reduce the craving for fatty and sweet foods, which could help in weight loss programmes (Giuffré et al., 2013). Studies have also suggested that stevia intake was able to reduce dietary intake of sugar and thus beneficial to obese individuals, diabetics and those with increased blood glucose levels (Thomas and Glade, 2010). Therefore, we could speculate that a longer duration of stevia intake, up to 4 weeks or more, could have shown a marked reduction of body weight and BMI. Recent systematic reviews compared the effects of stevia and other lowcalorie sweeteners with sucrose intake, satiety and body weight in healthy and obese individuals observed that stevia reduced the calorie intake of healthy and obese participants compared to those consuming sucrose (Ashwell, 2015; Rogers et al., 2016). Our participants were instructed to reduce or not consume any excess sucrose in their diet during the stevia and placebo intervention. A small reduction in weight and BMI was obtained that might be due the effects of stevia in reducing the glucose uptake in the small intestine (O'Brien-Nbors, 2011). In addition, all volunteers were instructed to maintain their minimum of $30 \mathrm{~min}$ of exercise every day and not to do vigorous exercise as this might affect their mean weight and this could signify a potential confounding factor. If a higher dose of stevia were given ( $1 \mathrm{~g}$ instead of $0.2 \mathrm{~g}$ of stevioside), the results might have shown a significant difference in weight compared to the baseline stevia or placebo intervention (Geeraert et al., 2010).

The dose of stevia consumed by our participants was small with ad libitum diet because we did not intend to restrict the habitual diet. However, there were few issues that have to be mentioned; firstly the amounts of stevia given in the sachets may not have been all consumed and therefore, there was no valid mean to confirm all participants were taking the correct dosage. Although stevia has been reported to be safe (Puri, 2012; Swithers, 2013), nevertheless, we did not want to use larger doses of stevia as the toxicity studies were not fully validated and the aim of study was explorative and short term. Secondly the stevia was a fine powder whereas the sugar placebo was crystallised, this may have had effects of recognition by the participants. Thirdly, a problem that a crossover study always faces was the carryover effect and the intervention period was only one week, so this problem could have been overcome by extending the washout period (Simpson et al., 2010). There was a high variance between participants and a small population that makes it difficult to draw any definite conclusions in the general population. This could perhaps be overcome with a more in depth inclusion and exclusion criteria and a larger number of volunteers. Future research should look into the effects of stevia consumption in different forms and preparations in both males and females from a range of different age groups, BMI and both hypertensive and normotensive individuals together with a cross-section population for longer and shorter periods of time. In addition, other physiological and biochemical parameters should be looked at such as fasting and postprandial blood glucose, insulin, gut and stress hormones. Moreover, examination of stevia consumption in diabetic patients would be useful to enhance our understanding of glucose metabolism and control that may occur over longer periods of time (Sattigeri, 2012).

\section{Conclusion}

This small study has shown that a short period of stevia intake caused a small but significant increase in BP and effects on body weight and BMI were not significant. The rise in BP could have been due to the increase in cortisol levels and modulation of $11 \beta$-HSD type 1 and 2 enzymes activity. Although further research in this area would be necessary, caution should be taken by the public who consume stevia for longer periods of time as a sweetener or other purposes. 


\section{Acknowledgment}

The authors are grateful for the technical help of staff at Queen Margaret University Biochemistry lab.

\section{Funding Information}

No specific funding from grant bodies or industry was offered to conduct this study.

\section{Author Contributions}

Emad A.S. Al-Dujaili: Has planned, executed and supervised the study. EASD, UA and FA have all contributed in writing the whole manuscript.

Husni Twaij and Yazan A. Bataineh: Have helped in the statistical analyses of data.

Unam Arshad and Faiza Amjid: Have recruited the volunteers, collected the samples and performed the physiological and anthropometry measures.

\section{Conflict of Interest}

The authors declare no conflict of interest.

\section{References}

Ahmed, B., M. Hossain, R. Islam, A.K. Saha and A. Mandal, 2011. A review on natural sweetener plant-stevia having medicinal and commercial importance. Prethodno Priopcenje.

Al-Dujaili, E.A.S., C.J. Kenyon, M.R. Nicol and J.I. Mason, 2011. Liquorice and glycyrrhetinic acid increase DHEA and deoxycorticosterone levels in vivo and in vitro by inhibiting adrenal SULT2A1 activity. Molecular Cellular Endocrinol., 336: 102-109.

Al-Dujaili, E.A.S., H.S. Baghdadi, F. Howie and J.I. Mason, 2012. Validation and application of a highly specific and sensitive ELISA for the estimation of cortisone in saliva, urine and in vitro cell-culture media by using a novel antibody. Steroids, 77: 703-709.

Anton, S.D., C.K. Martin and H. Han, 2010. Effects of stevia, aspartame and sucrose on food intake, satiety and postprandial glucose and insulin levels. Appetite, 55: 37-43.

Ashwell, M., 2015. Stevia, nature's zero-calorie sustainable sweetener: A new player in the fight against obesity. Nutrition Today, 50: 129-134.

Barriocanal, L.A., M. Palacios, G. Benitez, S. Benitez and J.T. Jimenez et al., 2008. Apparent lack of pharmacological effect of steviol glycosides used as sweeteners in humans. A pilot study of repeated exposures in some normotensive and hypotensive individuals and in Type 1 and Type 2 diabetics. Regul. Toxicol. Pharmacol., 51: 37-41. DOI: 10.1016/j.yrtph.2008.02.006
Bland, M., 2015. An Introduction to Medical Statistics. 3rd Edn., Oxford University Press, Oxford, ISBN-10: 0199589925, pp: 427.

Brahmachari, G., L.C. Mandal and R. Roy, 2011. Stevioside and related compounds-molecules of pharmaceutical promise: A critical overview. Arch. Pharm. (Weinheim), 344: 5-19.

Bryant, C.E., L.K. Wasse, N. Astbury, G. Nandra and J.T. McLaughlin, 2014. Non-nutritive sweeteners: No class effect on the glycaemic or appetite responses to ingested glucose. Eur. J. Clin. Nutriti., 68: 629-631.

Carakostas, M.C., L.L. Curry and A.C. Boileau, 2008. Overview: The history, technical function and safety of rebaudioside A, a naturally occurring steviol glycoside, for use in food and beverages. Food Chem. Toxicol., 46: S1-S10. DOI: 10.1016/j.fct.2008.05.003

Chan, P., B. Tomlinson and Y.J. Chen, 2000. A doubleblind placebo-controlled study of the effectiveness and tolerability of oral stevioside in human hypertension. Br J. Clin. Pharmacol., 50: 215-220.

Corcuff, J.B. and J. Brossaud, 2014. Rebaudioside A and cortisol metabolism: Sweet news for consumers. Clin. Achimicaacta., 431: 276-277.

Curry, L.L. and A. Roberts, 2008. Subchronic toxicity of rebaudioside A. Food Chem. Toxicol., 46: 11-20. DOI: $10.1016 /$ j.fct.2008.04.042

Delaney, E., 2014. The relationship between traumatic stress, PTSD and cortisol. Navy Med.

Edwards, C.R.W., 1991. Lessons from licorice. New England J. Med., 325: 1242-1243.

Esmail, S. and U.M. Kabadi, 2012. Edema, enigma: 11 B-hydroxysteroid dehydrogenase type 2 inhibition by sweetener "Stevia". J. Endocrine Metabol. Dis., 2: 49-52.

Ferri, L.A.F., A.P. Wilson, S.S. Yamada, S. Gazola and M.R. Batista et al., 2006. Investigation of the antihypertensive effect of oral crude stevioside in patients with mild essential hypertension. Phytother. Res., 20: 732-736.

Field, A., 2005. Discovering Statistics Using SPSS. 2nd Edn., London, Sage.

Franklin, S.S., L. Thijs, T.W. Hansen and J.A. Staessen, 2013. White-coat hypertension: New insights from recent studies. Hypertension, 2: 982-987.

Gardana, C., P. Simonetti, E. Canzi, R. Zanchi and P. Pietta, 2003. Metabolism of stevioside and rebaudioside A from Stevia rebaudiana extracts by human microflora. J. Agricultural Food Chem., 51: 6618-6622.

Geeraert, B., F. Crombe, M. Hulsmans, N. Benhabilès and J.M. Geuns et al., 2010. Stevioside inhibits atherosclerosis by improving insulin signaling and antioxidant defense in obese insulin-resistant mice. Int. J. Obes., 34: 569-577. DOI: 10.1038/ijo.2009.261

Geun, J.M., J. Buyse and A. Vankeirsbilck, 2007. Metabolism of stevioside by healthy subjects. Exp. Biol. Med. (Maywood), 232: 164-173. 
Gheisar, M.M. and I.H. Kim, 2014. Effect of dietary stevia on immune response of growing pigs challenged with escherichia coli lipopolysaccharide. Int. J. Agric. Innovations Res., 2: 1174-1177.

Giuffré, L., R. Romaniuk and E. Ciarlo, 2013. Stevia, ka'a he'e, wild sweet herb from South America: An overview. Emir. J. Food Agric., 25: 746-750.

Goyal, S.K. and R.K. Samsher, 2010. Stevia (Stevia rebaudiana) a bio-sweetener: A review. Int. J. Food Sci. Nutriti., 61: 1-10. DOI: $10.3109 / 09637480903193049$

Gregersen, S., P.B. Jeppesen and J.J. Holst, 2004. Antihyperglycemic effects of stevioside in type 2 diabetic subjects. Metabolism, 53: 73-76.

Gupta, E., S. Purwar, S. Sundaram and G.K. Rai, 2013. Nutritional and therapeutic values of Stevia rebaudiana: A review. J. Med. Plants Res., 7: 3343-3352.

Hsieh, M.H., P. Chan, Y.M. Sue, J.C. Liu and T.H. Liang et al., 2003. Efficacy and tolerability of oral stevioside in patients with mild essential hypertension: A two-year, randomized, placebocontrolled study. Clin. Therapeutics, 25: 2797-2808.

Hwang, K.O., K. Farheen, C.W. Johnson, E.J. Thomas and A.S. Barnes et al., 2007. Quality of weight loss advice on internet forums. Am. J. Med., 120: 604-609.

Jeppesen, P.B., S.E. Dyrskog and A. Agger, 2006. Can stevioside in combination with a soy-based dietary supplement be a new useful treatment of type 2 diabetes? An in vivo study in the diabetic gotokakizaki rat. Rev. Diabetes Stud., 3: 189-199.

Khalil, S.A., R. Zamir and N. Ahmad, 2014. Selection of suitable propagation method for consistent plantlets production in Stevia rebaudiana (Bertoni). Saudi J. Biol. Sci., 21: 566-573.

Lemus-Mondaca, R., A. Vega-Gálvez, L. Zura-Bravo and K. Ah-Hen, 2012. Stevia rebaudiana Bertoni, source of a high-potency natural sweetener: A comprehensive review on the biochemical, nutritional and functional aspects. Food Chem., 32: 1121-1132.

Maki, K.C., L.L. Curry and M.C. Carakostas, 2008. The hemodynamic effects of rebaudioside a in healthy adults with normal and low-normal blood pressure. Food Chem. Toxicol., 46: S40-S46.

NHS Choice, 2013. Addison's Disease [online].

O'Brien-Nbors, L., 2011. Alternative Sweeteners. 4th Edn., CRC Press, ISBN-10: 1439846154, pp: 587.

Onakpoya, I.J. and C.J. Heneghan, 2015. Effect of the natural sweetener, steviol glycoside, on cardiovascular risk factors: A systematic review and meta-analysis of randomised clinical trials. Europ. J. Preventive Cardiol., 22: 1575-87. DOI: $10.1177 / 2047487314560663$

Pallant, J., 2001. SPSS survival manual.
Pereira, C.D., I. Azevedo, R. Monteiro and M.J. Martins, 2012. $11 \beta$-hydroxysteroid dehydrogenase type 1 : Relevance of its modulation in the pathophysiology of obesity, the metabolic syndrome and type 2 diabetes mellitus. Diabetes Obes. Metab., 14: 869-81. DOI: $10.1111 /$ j.1463-1326.2012.01582.x

Puri, M., 2012. Extraction and safety of stevioside; response to the article "Stevia rebaudiana Bertoni, source of a high potency natural sweetener: A comprehensive review on the biochemical, nutritional and functional aspects. Food Chem., 1: 1861-1862. DOI: 10.1016/j.foodchem.2012.06.080

Quinkler, M. and P.M. Stewart, 2003. Hypertension and the cortisol-cortisone shuttle. J. Clin. Endocrinol. Metabolism, 88: 2392-2392.

Ranjan, R., J. Jaiswal and J. Jena, 2011. Stevia as a natural sweetener. Int. J. Res. Pharmacy Chem., 1: 1199-1202.

Rizzo, B., L. Zambonin and C. Angeloni, 2013. Steviol glycosides modulate glucose transport in different cell types. Oxid Med. Cell Longev. DOI: 10.1155/2013/348169.

Roberts, A. and I. Munro, 2009. Stevioside and related compounds: Therapeutic benefits beyond sweetness. Pharmacol. Ther., 122: 41-54. DOI: 10.1016/j.pharmthera.2009.03.005

Rogers, P.J., P.S. Hogenkamp, C. de Graaf, S. Higgs and A. Lluch et al., 2016. Does low-energy sweetener consumption affect energy intake and body weight? A systematic review, including metaanalyses, of the evidence from human and animal studies. Int. J. Obesity, 40: 381-394.

Sattigeri, B.M., 2012. Stevia -A natural low calorie sweetener with more promising benefits. Int. J. Phytothearpy Res., 2: 2278-2290.

Shivanna, N., M. Naika, F. Khanum and V.K. Kaul, 2013. Antioxidant, anti-diabetic and renal protective properties of Stevia rebaudiana. J. Diabetes Complicat., 27: 103-113.

Simpson, P.M., R.M. Hamer and S. Lesing, 2010. Cross crossover studies off your list.

Sorensen, L.B., T.H. Vasilaras, A. Astrup and A. Raben, 2014. Sucrose compared with artificial sweeteners: A clinical intervention study of effects on energy intake, appetite and energy expenditure after 10 wk of supplementation. Am. J. Clin. Nutr., 100: 36-45. DOI: 10.3945/ajcn.113.081554

Stewart, P.M., A.M. Wallace, S.M. Atherden, C. Shearing and C.R.W. Edwards, 1990. The mineralocorticoid activity of carbenoxolone: Contrasting effects of carbenoxolone and liquorice on 11ß-hydroxysteroid dehydrogenase activity in man. Clin. Sci., 78: 49-54. 
Swithers, S.E., 2013. Artificial sweeteners produce the counterintuitive effect of inducing metabolic derangements. Trends Endocrinology Metabolism, 24: 431-441.

Thomas, J.E. and M.J. Glade, 2010. Stevia: It's not just about calories. Open Obesity J., 2: 101-109.

Ulbricht, C., R. Isaac, T. Milkin, E.A. Poole and E. Rusie et al., 2010. An evidence-based systematic review of stevia by the natural standard research collaboration. Cardiovasc. Haematol. Agents Med. Chem., 8: 113-127.
Wheeler, A., A.C. Boileau and P.C. Winkler, 2008. Pharmacokinetics of rebaudioside $A$ and stevioside after single oral doses in healthy men. Food Chem. Toxicol., 46: S54-S60.

Whitworth, J.A., 2003. World Health Organisation (WHO)/International Society of Hypertension (ISH) statement of management of Hypertension. J. Hypertension, 21: 1983-1992.

Yadav, S.K. and P. Guleria, 2012. Steviol glycosides from stevia: Biosynthesis pathway review and their application in foods and medicine. Crit. Rev. Food Sci. Nutr., 52: 988-998. 